
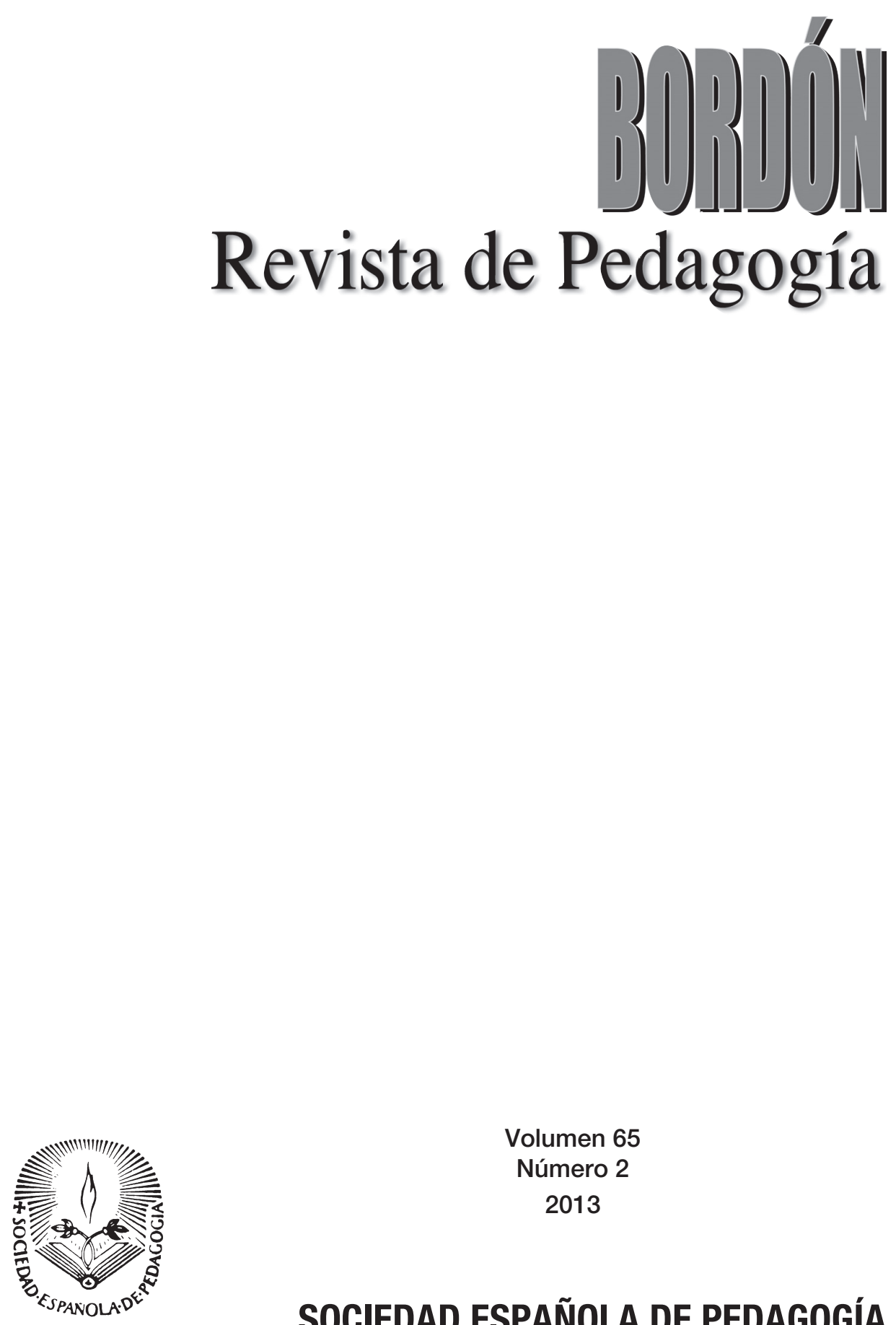

Volumen 65

Número 2

2013

SOCIEDAD ESPAÑOLA DE PEDAGOGÍA 



\title{
LA UTILIZACIÓn DEL JUICIO DE EXPERTO PARA LA EUALUACIÓn DE TIC: EL COEFICIERTE DE COMPETENCIA EXPERTA
}

\section{The Use of Expert Judgment for Assessing ICT: the Coefficient of Expert Competence}

\author{
JULIO CABERO ALMENARA \\ JULIO BARROSO OSUNA \\ Universidad de Sevilla
}

\begin{abstract}
INTRODUCCIÓN. El presente artículo trata sobre el uso de una de las técnicas o estrategias más utilizadas en las diferentes investigaciones en el terreno educativo: el juicio de expertos. Se analizan diferentes aspectos relacionados con el mismo, como la problemática de la conceptualización de los expertos, las ventajas y limitaciones que nos aporta su utilización en la investigación educativa, la diversidad de criterios que podemos movilizar para la selección de los expertos y el número adecuado de utilización de los mismos en una investigación. MÉTODO. Se presenta un proceso de selección de los expertos no muy usual en nuestra investigación educativa, el denominado «Coeficiente de competencia experta» o "Coeficiente K», que se obtiene a partir de la autovaloración realizada por la persona para determinar su competencia experta en la materia objeto de la investigación; más concretamente podemos decir que se obtiene mediante la aplicación de la siguiente fórmula: $\mathrm{K}=1 / 2(\mathrm{Kc}+\mathrm{Ka})$, donde $\mathrm{Kc}$ es el «Coeficiente de conocimiento» o información que tiene el experto acerca del tema o problema planteado; y Ka es el denominado «Coeficiente de argumentación» o fundamentación de los criterios de los expertos. RESULTADOS. Se ofrece un ejemplo de aplicación y obtención del mismo en una investigación financiada por el MEC, donde hemos diseñado un «Entorno personal de aprendizaje» (PLE) («Diseño, producción y evaluación de un entorno de aprendizaje 2.0 para la capacitación del profesorado universitario en la utilización educativa de las Tecnologías de la Información y Comunicación») (Dipro 2.0- EDU2009-08893). DISCUSIÓN. El trabajo presentado nos permite llegar a la conclusión de que el la «Técnica de Competencia Experta» nos permite discriminar de forma más adecuada la selección de los expertos.
\end{abstract}

Palabras clave: Evaluación, Tecnologías de la Información y la Comunicación, Juicio de expertos, Coeficiente de competencia experta.

\section{Algunas referencias iniciales}

La utilización de expertos como estrategia para la evaluación de materiales de enseñanza, de instrumentos de recogida y análisis de información, o de las metodologías empleadas es bastante usual en el terreno de la investigación educativa, y constituye, además, la base del método Delphi (Cañibano y Alberto, 2008; Barroso y Cabero, 2010; Romero et al., 2012). Presenta las siguientes ventajas: la teórica calidad de las respuestas alcanzadas, el nivel de profundización de las 
respuestas obtenidas, y el poder recoger una información pormenorizada (Cabero, 2001); al mismo tiempo se la considera como una técnica de gran utilidad para establecer el conocimiento del arte en contenidos y temáticas difíciles, complejas, novedosas o poco estudiadas (Shanteau, 1992; Lannoy y Procaccia, 2001).

En su aplicación uno de los problemas con que nos encontramos es el de la precisión de lo que entendemos por experto, pues el término en sí es bastante polisémico y, muchas veces, poco clarificador. También nos encontramos con el problema de su selección, respecto al que en los últimos tiempos están apareciendo nuevos procedimientos, como el denominado «Coeficiente de competencia experta», sobre el que nosotros nos centraremos en el presente trabajo, y presentaremos la forma en la que lo hemos aplicado en una investigación.

\section{Concepto de experto y procedimientos para su selección}

Como ya hemos señalado, uno de los problemas con que nos enfrentamos a la hora de la selección y concreción de los expertos es que su término es bastante polisémico y, como nos señala el Diccionario de la RAE, suele ir asociado a lo práctico, hábil o experimentado que podemos considerar a una persona. Como indica Mengual $(2011,158)$, podemos llegar a entender por experto: «tanto al individuo como al grupo de personas que son capaces de proporcionar valoraciones fiables sobre un problema en cuestión, y al mismo tiempo, hacen recomendaciones en función de un máximo de competencia». Por su parte Landeta $(2002,57)$ los define como «... aquel individuo cuya situación y recursos personales le posibiliten contribuir positivamente a la concesión del fin que ha motivado del trabajo Delphi».

Los criterios que se pueden utilizar para su selección son diversos (Brill et al., 2006; García y Fernández, 2008), y van desde la vinculación del experto con el problema, su experiencia profesional, sus cualidades personales para participar en las investigaciones o su pericia profesional. $\mathrm{Y}$ al respecto, posiblemente uno de los trabajos que más se ha extendido en aportar las diferentes características que deben poseer los expertos sea el realizado por Abdolhammadi y Shanteau $(1992,162)$, que nos ofrece las siguientes:

- Adaptabilidad: toman las decisiones adecuadas a la situación.

- Asunción de disponibilidad: aceptan los resultados que se alcancen.

- Creatividad: pueden encontrar una solución nueva o única a los problemas difíciles.

- Experiencia comunicativa: pueden convencer a otros de que poseen un mayor conocimiento del tema.

- Conocimiento actual: tienen una amplia base de conocimiento.

- Decisiones: toman decisiones de forma rápida, clara y enfáticamente. ${ }^{*}$ )

- Enérgico: son capaces de invertir mucha energía.

- Experiencia: tienen en cuenta su experiencia pasada.

- Inquisitivo: exhiben una amplia curiosidad para la resolución de las situaciones problemáticas. $(*)$

- Saber qué es relevante: discriminan la información relevante de la irrelevante.

- Hacer excepciones: saben cuándo el seguimiento estabiliza las estrategias y cuándo no.

- Metódico: se aproximan a las nuevas situaciones problemáticas de forma muy sistemática.

- Perceptivo: extraen información de los problemas que otros no saben.

- Perfeccionista: intentan logar niveles altos de toma de decisiones para adoptar la mejor de las posibles estrategias. (*)

- Apariencia física: presentan una imagen al exterior de ser los mejores para resolver los problemas. $(*)$

- Selectivo en los problemas: utilizan la prospectiva y prevén la planificación en la selección de los problemas. 
- Simplificación: saben cómo usar en investigación el «divide y vencerás» con problemas complejos.

- Confianza en uno mismo: tienen una fuerte creencia sobre su habilidad para tomar una buena decisión.

- Tolerante al estrés: toman decisiones en situaciones de alto estrés.

- Cálido y amigable: se llevan bien con las personas, incluso tomando decisiones difíciles. $(*)$

(Nota: ${ }^{*}=$ no son las relevantes).

\section{Aplicación de la estrategia del juicio de experto a la evaluación de TIC: Proyecto Dipro 2.0}

\section{Criterios iniciales utilizados para la selección de los expertos}

Nosotros en una investigación centrada en la creación de un «Entorno personal de aprendizaje» (PLE) («Diseño, producción y evaluación de un entorno de aprendizaje 2.0 para la capacitación del profesorado universitario en la utilización educativa de las Tecnologías de la Información y Comunicación» Dipro 2.0EDU2009-08893) (Cabero, Barroso y Llorente, 2010; Barroso, Cabero y Vázquez, 2012), perseguíamos una serie de objetivos entre los que se encuentran:

- Crear un entorno formativo telemático bajo la arquitectura web 2.0, destinado a la formación del profesorado universitario en la adquisición de diferentes capacidades y competencias para la utilización e inserción de las TIC en su actividad profesional.

- Validar el entorno formativo telemático, tanto en lo que se refiere a la propuesta de estructurar los contenidos, como a las diversas herramientas de comunicación (blog, wikis...) creadas.
Los entornos producidos, y que fueron objeto de la evaluación, pueden observarse en las siguientes direcciones web: http://tecnologiaedu.us.es/ portal/ y http://tecnologiaedu.us.es/dipro2/

Y para evaluarlo tomamos la decisión de que uno de los procedimientos que íbamos a utilizar para ello era el «juicio de experto» y establecimos los siguientes criterios iniciales para comenzar su selección, es decir, para comenzar la selección de los expertos que conformarían nuestra muestra:

- Tener experiencia laboral en el ámbito de la Tecnología Educativa, Nuevas Tecnologías aplicadas a la Educación, o Tecnologías de la Información y Comunicación Aplicadas a la Educación.

- Tener experiencia laboral en el ámbito de la formación virtual, e-learning, Tecnología Educativa, Nuevas Tecnologías aplicadas a la Educación, o Tecnologías de la Información y Comunicación Aplicadas a la Educación.

- Tener experiencia en formación virtual, e-learning y Entornos Personales de Aprendizaje.

- Tener una experiencia formativa superior a cinco años.

- Ser de diferentes Universidades Españolas.

- Ser de diferentes Universidades Latinoamericanas.

- El haber colaborado anteriormente en otros trabajos de investigación o de publicación, que nos permitiera realizar una evaluación previa de su seriedad y profesionalidad.

\section{Número de expertos a utilizar en una investigación}

Además de los criterios a utilizar para su selección, otro de los problemas con que nos encontramos en su aplicación es el del número de expertos necesarios para llevar a cabo un estudio (Williams y Webb, 1994; Powell, 2003). Y aquí 
nos hallamos con diferentes propuestas, como la de Malla y Zabala (1978) que nos sugiere que su número debe oscilar entre 15 y 20 ; la de Gordon (1994) que los sitúa entre 15-35; la de Landeta (2002) que indica que deben estar comprendido entre 7 y 30; García y Fernández (2008) para quienes el intervalo debe situarse entre 15 y 25; o la propuesta realizada por Witkin y Altschuld (1995) quienes no señalan un número concreto, pero sí nos llaman la atención de que debe ser menor que 50, reconociendo que en algunos casos pueden ser mayores.

Desde nuestro punto de vista, la selección del número de expertos depende muchas veces de diferentes aspectos, como son:

a) La posibilidad de poseer expertos suficientes con claras referencias hacia la temática analizada.

b) El evitar el menor número de pérdida de sujetos entre las dobles y triples vueltas que se hace necesario realizar en algunos estudios.

c) El volumen de trabajo que seamos capaces de mover.

d) La facilidad con que podamos acceder a los expertos.

e) La rapidez con que debamos ofrecer los resultados preliminares, sobre todo en estudios de varias vueltas, para evitar la desmotivación en la participación en el estudio de los «expertos», es decir, evitar la mortandad experimental.

En nuestro trabajo, una vez identificados los criterios de selección que hemos señalado anteriormente, nos pusimos en contacto con diferentes personas que creíamos que cumplían los criterios anteriores y que conocíamos por participación en eventos, colaboración en proyectos anteriores de investigación, o que teníamos alguna vinculación histórica con ellos. Comentar que, a la carta de invitación a participar en la investigación, nos contestaron 70, con los cuales inicialmente comenzamos nuestro trabajo.

\section{La puesta en acción del «Juicio de experto» en nuestra investigación: la obtención del «Coeficiente de competencia experta»}

Como es obvio, la calidad de los resultados que se consigan en un estudio donde apliquemos el «juicio de experto» va a estar completamente relacionada con los expertos seleccionados, de ahí que la utilización de un buen procedimiento de selección sea uno de los aspectos al que debamos prestar más atención en nuestro trabajo. Y como podemos imaginarnos, no existe una única forma de poner en acción un «juicio de expertos», $\mathrm{y}$ al respecto nos encontramos con diferentes posibilidades, siendo las más usuales las siguientes:

a) Agregación individual de los expertos, que consiste en obtener la información de manera individual de los diferentes expertos sin que ellos se encuentren en contacto.

b) Método Delphi, en el cual recogemos la opinión de los expertos de forma individual y anónima, devolviéndoles la propuesta de conjunto para su revisión y acuerdo; una débil dispersión nos llevará a señalar que se ha llegado a un acuerdo.

c) Técnica grupal nominal, en la que los expertos aportan su información de manera individual, y después de forma grupal y presencial se llega a un acuerdo.

d) Método de consenso, donde de forma grupal y de conjunto los expertos seleccionados llegan a establecer un acuerdo.

Lógicamente, la selección de uno de estos procedimientos dependerá de diferentes aspectos, como la rapidez con que el investigador deba llevar a cabo el estudio, la profundización de los resultados que desee conseguir y su homogeneidad.

Para su selección los investigadores suelen limitarse a la construcción de un biograma del experto, a partir de la formulación de una serie de preguntas respecto a sus años de trabajo, volumen de producción científica, cargos que 
ha ocupado, titulación...; y desde sus contestaciones en las mismas, se ha inferido la «calidad»y «validez» del experto seleccionado, y su incorporación a la investigación.

Sin dejar de señalar que el procedimiento mencionado anteriormente no debe ser radicalmente eliminado, y puede perfectamente combinarse con el que nosotros después vamos a presentar. Tenemos que remarcar que en los últimos tiempos están apareciendo diferentes tipos de técnicas centradas en la autovaloración de la persona para determinar su competencia experta, que pueden permitirnos obtener expertos más significativos para nuestros trabajos. Uno de estos procedimientos es el denominado «Coeficiente de competencia experta» o «Coeficiente K» (Oñate, 2001, García y Fernández, 2008; López, 2008; García y Fernández, 2008; Blasco et al., 2010).

\section{Tabla 1. Valoración de las fuentes de argumentación para la obtención de «Coeficiente de Argumentación» (Ka)}

\begin{tabular}{|c|c|c|c|}
\hline \multirow[t]{2}{*}{$\begin{array}{l}\text { Fuente de } \\
\text { argumentación }\end{array}$} & \multicolumn{3}{|c|}{$\begin{array}{l}\text { Grado de influencia } \\
\text { de cada una de las fuentes } \\
\text { en sus criterios }\end{array}$} \\
\hline & $\begin{array}{c}\text { A } \\
\text { (alto) }\end{array}$ & $\begin{array}{c}\text { M } \\
\text { (Medio) }\end{array}$ & $\begin{array}{c}\text { B } \\
\text { (bajo) }\end{array}$ \\
\hline $\begin{array}{l}\text { Análisis teóricos } \\
\text { realizados por el } \\
\text { experto }\end{array}$ & 0,3 & 0,2 & 0,1 \\
\hline Experiencia obtenida & 0,5 & 0,4 & 0,2 \\
\hline $\begin{array}{l}\text { Estudio de trabajos } \\
\text { sobre el tema, } \\
\text { de autores españoles } \\
\end{array}$ & 0,05 & 0,05 & 0,05 \\
\hline $\begin{array}{l}\text { Estudio de trabajos } \\
\text { sobre el tema, de } \\
\text { autores extranjeros }\end{array}$ & 0,05 & 0,05 & 0,05 \\
\hline $\begin{array}{l}\text { Conocimiento propio } \\
\text { acerca del estado } \\
\text { del problema } \\
\text { en el extranjero }\end{array}$ & 0,05 & 0,05 & 0,05 \\
\hline Intuición del experto & 0,05 & 0,05 & 0,05 \\
\hline
\end{tabular}

El «Coeficiente K» está siendo utilizado en los últimos tiempos en diferentes estudios referidos a distintas problemáticas, y así nos encontramos con los trabajos de: Cuesta y Godinez, (2008); López et al. (2011 y 2012); Góngora et al. (2009); González et al. (2010); González et al. (2009); Herrera et al. (2010); Mengual (2011) y Zayas (2011). Nosotros también lo hemos aplicado en un reciente trabajo con resultados significativos, y por ello vamos a explicar a continuación cómo lo obtuvimos.

El cálculo del «Coeficiente de competencia experta» se efectúa a partir de la opinión mostrada por el experto sobre su nivel de conocimiento acerca del problema de investigación, así como de las fuentes que le permiten argumentar el criterio establecido, realizándose a través de las preguntas que presentamos en el Anexo.

El coeficiente se obtiene mediante la aplicación de la siguiente fórmula:

$$
K=1 / 2(K c+K a)
$$

donde:

$\mathrm{Kc}=$ Es el «Coeficiente de conocimiento»o información que tiene el experto acerca del tema o problema planteado. Es calculado a partir de la valoración que realiza el propio experto en la escala del 0 al 10, multiplicado por 0,1.

$\mathrm{Ka}=$ Es el denominado «Coeficiente de argumentación» o fundamentación de los criterios de los expertos. Este coeficiente se obtiene a partir de la asignación de una serie de puntuaciones a las distintas fuentes de argumentación que ha podido esgrimir el experto. En la tabla 1 , se ofrecen las puntuaciones usualmente utilizadas para la valoración de las fuentes de argumentación, de acuerdo con los diferentes autores (Oñate, 2001; García y Fernández, 2008; López, 2008; García y Fernández, 2008; Blasco et al., 2010) que han desarrollado el procedimiento, lógicamente, en función de la contestación del experto. 
Con los valores finales obtenidos se clasifican los expertos en tres grandes grupos:

- Si K es mayor a 0,8, mayor o menor o igual a 1: entonces hay influencia alta de todas las fuentes.

- Si K es mayor o igual que 0,7, mayor o menor o igual a 0,8: entonces hay influencia media de todas las fuentes

Si $\mathrm{K}$ es mayor o igual a 0,5 , mayor o menor o igual a 0,7 entonces hay influencia baja de todas las fuentes.

Tenemos que señalar que, de acuerdo con la filosofía de la propuesta de obtención del «Coeficiente de competencia experta» (Oñate, 2001; García y Fernández, 2008; López, 2008; García y Fernández, 2008; Blasco et al., 2010; Mengual, 2011), aquellos expertos analizados que hubieran obtenido valores inferiores a 0,8 no son contemplados y, por lo tanto, rechazados en el estudio.

Como ya hemos señalado anteriormente, en nuestra investigación incorporamos dos preguntas con las que obtener el "Coeficiente experto» (Anexo). La primera de ellas perseguía recoger información respecto a la autovaloración que los expertos se hacían, en una escala de 0 a 10, del grado de conocimiento que creían tener acerca de temáticas como las siguientes: formación virtual, formación a distancia, e-learning o entornos de aprendizaje; es decir, sobre aspectos relacionados con nuestra temática de estudio. Lógicamente el valor $« 0$ » indicaba absoluto desconocimiento de la problemática que se evalúa, mientras que su opuesto, el valor «10», hacía referencia al pleno conocimiento de la referida problemática.

La puntuación media que se obtuvo con los 70 expertos que inicialmente participaron en la investigación fue de 7,91, con una desviación típica del 1,64. Puntuaciones que nos permitían señalar que nuestros expertos consideraban que tenían una aceptable información respecto a la temática sobre la que giraba nuestra investigación.

La otra pregunta perseguía la obtención del «Coeficiente de argumentación» y proponía que el experto valorara el grado de influencia que una serie de fuentes habían tenido en el conocimiento que poseía respecto a la formación virtual, formación a distancia, e-learning, entornos personales de aprendizaje... En la tabla 2, presentamos los valores medios y las desviaciones típicas alcanzadas; para su correcta interpretación téngase en cuenta las tres opciones que se le ofrecían al sujeto: baja (1), media (2) y alta (3).

TABla 2. Valoración de diferentes fuentes por el experto para la construcción del conocimiento respecto a la formación virtual, formación a distancia, e-learning, entornos personales de aprendizaje... Medias y desviaciones típicas

Fuente

\begin{tabular}{lll} 
Análisis teóricos realizados por usted & 2,44 & 0,694 \\
\hline Su experiencia obtenida de su actividad práctica & 2,81 & 0,392 \\
\hline Estudio de trabajos sobre el tema, de autores españoles & 2,57 & 0,650 \\
\hline Estudio de trabajos sobre el tema, de autores extranjeros & 2,34 & 0,611 \\
\hline $\begin{array}{l}\text { Su propio conocimiento acerca del estado del problema } \\
\text { en el extranjero }\end{array}$ & 1,94 & 0,679 \\
\hline Su intuición sobre el tema abordado & 2,29 & 0,684 \\
\hline
\end{tabular}


TABla 3. Valoración de diferentes fuentes por el experto para la construcción del conocimiento respecto a la formación virtual, formación a distancia, e-learning, entornos personales de aprendizaje... Frecuencias y porcentajes

\begin{tabular}{lcccccc} 
& \multicolumn{2}{c}{ Bajo } & \multicolumn{2}{c}{ Medio } & \multicolumn{2}{c}{ Alto } \\
\cline { 2 - 7 } & $\mathrm{f}$ & $\%$ & $\mathrm{f}$ & $\%$ & $\mathrm{f}$ & $\%$ \\
\hline & 8 & 11,4 & 23 & 32,9 & 39 & 55,7 \\
\hline $\begin{array}{l}\text { Análisis teóricos realizados por usted } \\
\text { Su experiencia obtenida de su actividad práctica }\end{array}$ & 0 & 0 & 13 & 18,6 & 5 & 81,4 \\
\hline $\begin{array}{l}\text { Estudio de trabajos sobre el tema, de autores } \\
\text { españoles }\end{array}$ & 6 & 8,6 & 18 & 25,7 & 46 & 65,7 \\
$\begin{array}{l}\text { Estudio de trabajos sobre el tema, de autores } \\
\text { extranjeros }\end{array}$ & 5 & 7,1 & 36 & 51,4 & 29 & 41,4 \\
$\begin{array}{l}\text { Su propio conocimiento acerca del estado } \\
\text { del problema en el extranjero }\end{array}$ & 18 & 25,7 & 38 & 54,3 & 14 & 20,0 \\
\hline $\begin{array}{l}\text { Su intuición sobre el tema abordado } \\
\text { n }\end{array}$ & 9 & 12,9 & 32 & 45,7 & 29 & 41,4 \\
\hline
\end{tabular}

En la tabla 3 presentamos las frecuencias y porcentajes alcanzados para cada una de las fuentes ofrecidas anteriormente.

Como ya hemos señalado estas dos preguntas son las tradicionalmente utilizadas para obtener el «Coeficiente de competencia experta» (Oñate et al., 1988; Oñate, 2001; López, 2008; García y Fernández, 2008; Blasco et al., 2010); aunque por supuesto deben adaptarse a la problemática del trabajo que estemos realizando.

El análisis de las dos tablas citadas anteriormente nos permite señalar que los 70 expertos, de acuerdo a los criterios expuestos al inicio de nuestro trabajo, mostraban unos altos niveles de argumentación respecto al conocimiento que tienen sobre las temáticas que en nuestro caso se relacionaban con la formación a distancia, virtual, el e-learning y los entornos personales de aprendizaje.

Una vez obtenidos el «Coeficiente de conocimiento» (Kc) y el «Coeficiente de argumentación» (Ka), mediante el procedimiento descrito anteriormente, pasamos a concretar el «Coeficiente de competencia experta», obtenido mediante la formulada expuesta en su momento. En la tabla 4, ofrecemos los valores alcanzados en los diferentes coeficientes mencionados de 70 expertos a los que inicialmente les enviamos el cuestionario de valoración del entorno.

Como podemos observar por los valores señalados en la tabla 4, y de acuerdo con el criterio de no contemplar a aquellos cuyo valor alcanzado en el «Coeficiente de competencia experta» fuese inferior a 0,8, nos llevó a eliminar a 17 de ellos, y por tanto a quedarnos con 53 expertos para la evaluación de nuestros entornos tecnológicos.

Con objeto de indagar si las respuestas ofrecidas por los expertos con «Coeficiente de consistencia» superior e inferior al 0,8 discriminaban las evaluaciones que realizaban los expertos respecto a los entornos tecnológicos producidos, aplicamos la prueba U de Mann-Whitney (Siegel, 1976; Pardo y Ruiz, 2005), para contrastar las siguientes hipótesis:

- Ho (Hipótesis Nula): No hay diferencias significativas, con un riesgo alfa de equivocarnos, entre las opiniones que ofrecieron los expertos que mostraban un 
«Coeficiente K» inferior a 0,8 y los que tenían puntuaciones superiores a la misma.

- H1 (Hipótesis Alternativa): Sí hay diferencias significativas, con un riesgo alfa de equivocarnos, entre las opiniones que ofrecieron los expertos que mostraban un «Coeficiente K» inferior a 0,8 y los que tenían puntuaciones superiores a la misma.

Tabla 4. Coeficiente de conocimiento (Kc), Coeficiente de argumentación (Ka) y Coeficiente de competencia experta (K), obtenidos por cada uno de los expertos

Coeficiente de conocimiento $(\mathrm{Kc})$
Coeficiente de argumentación (Ka)
Coeficiente de competencia experta (K)

\begin{tabular}{|c|c|c|c|}
\hline 1 & 0,70 & 0,80 & 0,75 \\
\hline 2 & 0,70 & 0,80 & 0,75 \\
\hline 3 & 0,50 & 0,70 & 0,60 \\
\hline 4 & 0,20 & 0,70 & 0,45 \\
\hline 5 & 0,50 & 0,80 & 0,65 \\
\hline 6 & 0,50 & 1,00 & 0,75 \\
\hline 7 & 0,60 & 0,80 & 0,70 \\
\hline 8 & 0,50 & 0,70 & 0,60 \\
\hline 9 & 0,60 & 0,80 & 0,70 \\
\hline 10 & 0,60 & 0,80 & 0,70 \\
\hline 11 & 0,30 & 0,80 & 0,55 \\
\hline 12 & 0,80 & 0,70 & 0,75 \\
\hline 13 & 0,60 & 0,70 & 0,65 \\
\hline 14 & 0,80 & 1,00 & 0,90 \\
\hline 15 & 0,80 & 1,00 & 0,90 \\
\hline 16 & 0,80 & 1,00 & 0,90 \\
\hline 17 & 0,90 & 1,00 & 0,95 \\
\hline 18 & 0,80 & 1,00 & 0,90 \\
\hline 19 & 0,80 & 0,90 & 0,85 \\
\hline 20 & 0,70 & 0,90 & 0,80 \\
\hline 21 & 0,90 & 1,00 & 0,95 \\
\hline 22 & 0,90 & 1,00 & 0,95 \\
\hline 23 & 0,80 & 0,90 & 0,85 \\
\hline 24 & 0,90 & 1,00 & 0,95 \\
\hline 25 & 0,90 & 1,00 & 0,95 \\
\hline 26 & 0,90 & 1,00 & 0,95 \\
\hline 27 & 0,90 & 1,00 & 0,95 \\
\hline 28 & 0,80 & 0,90 & 0,85 \\
\hline 29 & 0,90 & 0,90 & 0,90 \\
\hline 30 & 0,80 & 1,00 & 0,90 \\
\hline 31 & 0,80 & 0,90 & 0,85 \\
\hline 32 & 0,90 & 1,00 & 0,95 \\
\hline 33 & 0,70 & 1,00 & 0,85 \\
\hline 34 & 1,00 & 1,00 & 1,00 \\
\hline 35 & 0,80 & 1,00 & 0,90 \\
\hline 36 & 0,80 & 0,90 & 0,85 \\
\hline
\end{tabular}


Tabla 4. Coeficiente de conocimiento (Kc), Coeficiente de argumentación (Ka) y Coeficiente de competencia experta (K), obtenidos por cada uno de los expertos (cont.)

Coeficiente de conocimiento $(\mathrm{Kc})$
Coeficiente de argumentación (Ka)
Coeficiente de competencia experta (K)

\begin{tabular}{|c|c|c|c|}
\hline 37 & 0,70 & 0,90 & 0,80 \\
\hline 38 & 0,60 & 1,00 & 0,80 \\
\hline 39 & 0,70 & 0,90 & 0,80 \\
\hline 40 & 0,80 & 0,90 & 0,85 \\
\hline 41 & 0,80 & 1,00 & 0,90 \\
\hline 42 & 0,80 & 0,80 & 0,80 \\
\hline 43 & 0,90 & 0,90 & 0,90 \\
\hline 44 & 0,80 & 1,00 & 0,90 \\
\hline 45 & 0,90 & 1,00 & 0,95 \\
\hline 46 & 0,80 & 1,00 & 0,90 \\
\hline 47 & 0,90 & 1,00 & 0,95 \\
\hline 48 & 1,00 & 1,00 & 1,00 \\
\hline 49 & 1,00 & 0,90 & 0,95 \\
\hline 50 & 0,60 & 1,00 & 0,80 \\
\hline 51 & 0,70 & 1,00 & 0,85 \\
\hline 52 & 0,90 & 0,90 & 0,90 \\
\hline 53 & 0,80 & 0,90 & 0,85 \\
\hline 54 & 1,00 & 1,00 & 1,00 \\
\hline 55 & 0,90 & 0,90 & 0,90 \\
\hline 56 & 0,90 & 1,00 & 0,95 \\
\hline 57 & 0,90 & 0,70 & 0,80 \\
\hline 58 & 1,00 & 1,00 & 1,00 \\
\hline 59 & 0,80 & 1,00 & 0,90 \\
\hline 60 & 1,00 & 1,00 & 1,00 \\
\hline 61 & 0,90 & 1,00 & 0,95 \\
\hline 62 & 0,90 & 1,00 & 0,95 \\
\hline 63 & 0,70 & 1,00 & 0,85 \\
\hline 64 & 0,90 & 0,90 & 0,90 \\
\hline 65 & 1,00 & 1,00 & 1,00 \\
\hline 66 & 0,80 & 1,00 & 0,90 \\
\hline 67 & 1,00 & 1,00 & 1,00 \\
\hline 68 & 1,00 & 1,00 & 1,00 \\
\hline 69 & 0,90 & 0,90 & 0,90 \\
\hline 70 & 0,70 & 0,90 & 0,80 \\
\hline
\end{tabular}

En la tabla 5, presentamos las puntuaciones alcanzadas en las tres partes que conformaban el cuestionario que específicamente realizamos en la investigación para la evaluación del «Entorno personal de aprendizaje» producido. 
TAbla 5. Valores de los rangos alcanzados ( $R P=$ Rango Promedio; $S M=$ Suma de Rangos)

\begin{tabular}{lllll} 
Nivel de competencia & N & RP & SM \\
\hline \multirow{2}{*}{ Bloque A } & Nivel Medio $(<0,8)$ & 13 & 25,77 & 335,00 \\
\cline { 2 - 4 } & Nivel Alto $(>=0,8)$ & 57 & 37,72 & 2150,50 \\
\cline { 2 - 4 } & Total & 70 & 30,73 & 399,00 \\
\hline Bloque B & Nivel Medio $(<0,8)$ & 13 & 36,59 & 2085,50 \\
\cline { 2 - 4 } & Nivel Alto $(>=0,8)$ & 57 & & 410,50 \\
\hline Bloque C & Notal & 70 & 31,58 & 2074,50 \\
\cline { 2 - 4 } & Nivel Medio $(<0,8)$ & 13 & 36,39 & \\
\cline { 2 - 4 } & Total Alto $(>=0,8)$ & 57 & &
\end{tabular}

TABla 6. U de Mann-Whiney para los tres bloques. $(*=$ significativo al 0,05$)$

\begin{tabular}{lcccc} 
& U de Mann-Whitney & W de Wilcoxon & Z & Sig. \\
\hline Bloque A & 244,000 & 335,000 & $-1,912$ & $0,05(*)$ \\
\hline Bloque B & 308,500 & 399,500 &,- 0945 & 0,34 \\
\hline Bloque C & 319,500 & 410,500 & $-0,773$ & 0,43 \\
\hline
\end{tabular}

En primer lugar en la tabla 6 presentamos las puntuaciones alcanzadas en las tres partes del cuestionario.

Los resultados obtenidos nos permiten rechazar la $\mathrm{HO}$ y aceptar la $\mathrm{H} 1$, únicamente para el bloque $\mathrm{A}$, con un riesgo alfa de equivocarnos del 0,05. En consecuencia, podemos concluir que únicamente en este bloque se han dado diferencias significativas entre las valoraciones que realizaron los expertos con un «Coeficiente $K »$ superior a 0,8 e inferior a dicha puntuación. De todas formas, podemos también señalar que los valores negativos obtenidos en las puntuaciones $\mathrm{Z}$ nos sugieren que las valoraciones de los expertos con coeficiente superior a 0,8 son mayores a las mostradas por los otros expertos.

De cualquier forma, nos gustaría señalar que, incluso en los dos bloques donde no se han rechazado las $\mathrm{H} 0$, los valores asignados por los expertos con «Coeficiente $K$ » superior a 0,8 son mayores que las asignadas por sus compañeros. Aspectos que también podemos observar por el signo negativo alcanzado en los valores $\mathrm{Z}$.

\section{Conclusiones y discusión de los resultados}

El trabajo que hemos presentado nos permite llegar a una serie de conclusiones, la primera de ella es que la técnica del «Coeficiente de competencia experta» nos permite discriminar de forma más adecuada la selección de los expertos, pues no nos apoyamos únicamente en un biograma realizado sobre los jueces a partir de preguntas sino de autovaloraciones más específicas que realiza el juez sobre su competencia para llevar a cabo la acción que se le va a solicitar. 
Por otra parte, creemos que tanto el procedimiento del biograma y el coeficiente pueden combinarse, utilizando en primer lugar el biograma para una preselección de los expertos y posteriormente el «Coeficiente de competencia experta» para el afinamiento.
Por último, señalar que creemos conveniente seguir trabajando en el aspecto metodológico utilizado para la obtención del «Coeficiente de competencia experta».

\section{Anexo}

\section{Preguntas formuladas para la obtención de «Coeficiente de competencia experta»}

1) Marque en la casilla que le corresponde el grado de conocimiento que usted posee acerca de temáticas como las siguientes: formación virtual, formación a distancia, e-learning, o entornos personales de aprendizaje. Valorándose en una escala de 0 a 10 (considerando el 0 como no tener absolutamente ningún conocimiento y 10 de pleno conocimiento del estado de la cuestión).

\begin{tabular}{|l|l|l|l|l|l|l|l|l|l|}
\hline 0 & \multicolumn{5}{|c|}{10} & 10 \\
\hline & & & & & & & & & \\
\hline
\end{tabular}

2) Autovalore el grado de influencia que cada una de las fuentes que le presentamos a continuación ha tenido en su conocimiento y criterios sobre el tema de desarrollo de la formación virtual, formación a distancia, e-learning, entornos personales de aprendizaje.

Bajo Medio Alto

Análisis teórico realizado por usted

Su experiencia obtenida de su actividad práctica

Estudio de trabajos sobre el tema, de autores españoles

Estudio de trabajos sobre el tema, de autores extranjeros

Su propio conocimiento acerca del estado del problema en el extranjero

Su intuición sobre el tema abordado

\section{Referencias bibliográficas}

Abdolhammadi, M. y Shanteau, J. (1992). «Organizational behavior and human decision processes». Personal attributes of expert auditors, 53 (2), 158-172.

Barroso, J. y Cabero, J. (2010). La investigación educativa en TIC. Visiones prácticas. Madrid: Síntesis.

Barroso, J.; Cabero, J. y VÁzquez, A. (2012). Formación desde la perspectiva de los entornos personales de aprendizaje. Apertura. http://www.udgvirtual.udg.mx/apertura/num16/1_formacion. html (20/07/2012). 
Blasco, J. E. et al. (2010). Validación mediante el método Delphi de un cuestionario para conocer las experiencias e interés hacia las actividades acuáticas con especial atención al windsurf. Ágora para la educación física y le deporte, 12 (1), 75-94.

BriLl, J.M. et al. (2006). The competencies and characteristics required of an effective project manager: A web-based Delphi study. Education Technology Research, 54 (2), 115-140.

CABEro, J. (2001). Tecnología educativa. Diseño y utilización de medios en la enseñanza. Barcelona: Paidós.

CABERo, J. (dir.) (2006). Formación del profesorado universitario en estrategias metodológicas para la incorporación del aprendizaje en red en el Espacio Europeo de Educación Superior. Píxel-Bit. Revista de Medios y Educación, 27, 11-29.

Cabero, J.; Barroso, J. y Llorente, M. C. (2010). El diseño de Entornos Personales de Aprendizaje y la formación de profesores en TIC. Digital Education Review, 18, 26-37 (http://greav.ub.edu/der/ index.php/der/article/view/169/298) (20/07/2012).

Cañibano, L. y Alberto, F. (2008). El control instruccional de la información financiera: aplicación de un estudio Delphi. Revista Española de Financiación y Contabilidad, 37 (140), 795-829.

Cuesta, M. y Godinez, C. (2008): Metodología para la mejora de los procesos del sistema de gestión de la calidad de la gerencia de proyectos de ETECSA. Industrial, XXIX, 2, 1-7.

García, L. y Fernández, S. (2008). Procedimiento de aplicación del trabajo creativo en grupo de expertos. Energética, XXIX (2), 46-50.

GÓNGORA, C. et al. (2009). Propuesta de competencias laborales para médicos que brindan servicios de atención médica en hoteles cubanos. Educación Médica Superior, 23 (3), http://scielo.sld.cu/ scielo.php?script=sci_arttext\&pid=S0864-21412009000300002 (8/06/2012).

GonZÁlez, B. et al. (2010). Sistema de acciones para el perfeccionamiento de la cultura general en estudiantes del primer año de Medicina Transfusional. MediSur, 8 (6), 32-38.

GonZÁlez, L. et al. (2009). Factores para evaluar la viabilidad de proyectos de conservación de edificaciones esenciales, no productivas, en zonas sísmicas. Ingeniería, Revista Académica de la FI-UADY, 13 (1), 25-39.

Gordon, T. J. (1994). The Delphi method, http://www.futurovenezuela.org/_curso/5-delphi.pdfS.

Herrera, M. et al. (2010). Análisis de los factores que inciden en el desgaste de los órganos de trabajo de los aperos de labranza. Revista Ciencias Técnicas Agropecuarias, 19 (2), http://scielo.sld.cu/ scielo.php?pid=S2071-00542010000200001\&script=sci_arttext (8/06/2012).

LANDETA, J. (2002). El método Delphi: una técnica de previsión del futuro. Barcelona: Ariel.

Lannoy, A. y Porcaccia, M. (2001). L'utilisation du jugement d'experts en sûreté de fonctionnement. Pais: Editions TEC \& DOC.

López, A. (2008). La moderación de la habilidad diagnóstico patológico desde el enfoque histórico cultural para la asignatura Patología Veterinaria. Revista Pedagógica Universitaria, 13 (5), 51-71.

López, C. et al. (2011). Establecimiento de conceptos básicos para una Educación Física saludable a través del Método Experto. Revista Electrónica de Investigación Educativa, 13 (2), 22-40.

López, C. et al. (2012). Conocimientos básicos de Educación Física-salud para autogestión de la Actividad Física. Revista Iberoamericana de Educación / Revista Ibero-americana de Educação, 58 (2), 1-10. (http://www.rieoei.org/deloslectores/4629Lopez.pdf) (20/06/2012).

Malla, F. y Zabala, I. (1978). La previsión del futuro en la empresa (III): el método Delphi. Estudios Empresariales, 39, 13-24.

Mengual, S. (2011). La importancia percibida por el profesorado y el alumnado sobre la inclusión de la competencia digital en educación Superior. Alicante: Departamento de Didáctica General y Didácticas específicas de la Facultad de Alicante.

OÑATE, M. (2001). Utilización del método Delphy en la pronosticación: Una experiencia inicial. Aplicación del Método Delphy, 2001. www.rieoei.org/deloslectores/804Bravo. 
Pardo Merino, A. y Ruiz, M. A. (2005). Análisis de datos con SPSS 13 base. Madrid: Mc Graw-Hill. Powell, C. (2003). The Delphi technique: myths and realities. Journal of Advanced Nursing, 41 (4), 376-382.

Romero, R. et al. (2012). El método Delphi y la formación del profesorado en TIC. Global, 9 (44), 81-92.

Shanteau, J. (1992). How much information does an expert use? Is it relevant? Acta Psychologica, $81,75-86$.

SIEGEL, S. (1976). Estadística no paramétrica. México: Trillas.

Williams, P. L. y WebB, C. (1994). The Delphi technique: A methodological discussion. Journal of Advanced Nursing, 19, 180-186.

Witkin, B. R. y Altschuld, J. W. (1995). Planning and conducting needs assessment: A practical guide. Thousand Oaks, Sage.

ZAYAS, P. (2011). El proceso del análisis y la descripción con las especificaciones para confeccionar la matriz de las competencias y construir el perfil del cargo o de ocupación. Ejemplo de dependiente gastronómico en la rama turística. TURyDES. Revista de Investigación y Desarrollo Local, 4 (9). http://www.eumed.net/rev/turydes/09/pmza.htm (8/06/2012).

\section{Abstract}

The Use of Expert Judgment for Assessing ICT: the Coefficient of Expert Competence

INTRODUCTION. This article discusses the use expert judgement, one of the techniques or strategies that is most widely used by research studies in the field of education. It also analyzes different aspects related to expert judgement, ranging from the problem of conceptualization of the experts, the advantages and limitations related to its use in educational research, the range of criteria that can be applied to select experts, and the ideal number of experts for a research study. METHODS. More specifically we present a selection process for experts which is not very common in our educational research, the so-called expert competence coefficient or coefficient «K», which is obtained from someone's self-assessment to determine his or her expert competence in the area of expertise being considered, This is obtained by applying the following formula: $K=1 / 2(\mathrm{Kc}+\mathrm{Ka})$, where $\mathrm{Kc}=$ the «knowledge coefficient» or information that the expert possesses regarding the subject or problem; and $\mathrm{Ka}=$ the «argument coefficient» or basis for the criteria for the expert. RESULTS and DICUSSION. A practical application of this model in a research study funded by the MEC is outlined, where we have designed a «personal learning environment» (PLE) («Design, Production and Evaluation of a 2.0 Learning Environment for the Training of University Teachers in the Educational Use of Information and Communication Technology« (2.0-EDU2009 Dipro-08893).

Key words: Assessment, Information and Communication Technologies, Expert Judgment, Expert Competence Factor.

\section{Résumé}

\section{L'utilisation de jugementd'expertpourévaluer les TIC: le coefficient de compétencetechnique}

Cet article traite de l'utilisation des techniques ou des stratégiesutiliséesdans les enquêtesdifférentesdans le domaine de l'éducation: un jugementd'expert. Dans la mêmeanalyse de différentsaspectsliés à elle, allant 
de la conceptualisation de problème de les experts, les avantages et les limites quirendent son utilisationdans la recherche en éducation, la diversité des approches que nouspouvonsmobiliserpour la sélection des experts, et le nombre approprié de celui-ci l'utilisationdans une enquête. Plus précisément, nousprésentons un processus de sélection des expertsn'estpastrèshabituelsdansnotrerecherche en éducation, le coefficient de soi-disantexpert de compétenceou le coefficient «K», quiestobtenu à partir de l'auto-évaluationpar la personneafin de déterminerleurcompétenced'expertdans le objet de l'enquête, plus précisément, nouspouvonsdire que l'onobtient en appliquant la formule suivante: $\mathrm{K}=1 / 2(\mathrm{Kc}+\mathrm{Ka})$, où $\mathrm{Kc}=\mathrm{le}$ "coefficient de la connaissance» ou de l'information que l'expert sur le sujetouproblème, et $\mathrm{Ka}=$ est le «coefficient de l'argument» ou de la fondation des critèrespour les experts. Un exempled'application et de l'obtenirdans une recherchefinancée par le MEC, oùnousavonsconçu un «environnementd'apprentissagepersonnel» (PLE) («Conception, réalisation et évaluationd'unenvironnementd'apprentissage 2.0 pour la formation des professeursd'université sur l'utilisationpédagogique des technologies de l'information et de la Communication «(2,0-EDU2009 Dipro-08893).

Mots clés: Évaluation, technologies de l'information et de communication, un jugementd'expert, le facteur de la compétenced'experts.

\section{Perfil profesional de los autores}

\section{Julio Cabero Almenara}

Catedrático de Tecnología Educativa de la Universidad de Sevilla, Director de Secretariado de Recursos Audiovisuales y Nuevas Tecnologías de la Universidad de Sevilla, Director del Grupo de Investigación Didáctica. Ha publicado diferentes trabajos en revistas especializadas sobre Tecnología Educativa. Ha impartido conferencias en diferentes Universidades españolas y latinoamericanas. Pueden encontrarse más referencias del autor en http://tecnologiaedu.us.es

Correo electrónico de contacto: cabero@us.es

\section{Julio Barroso Osuna}

Profesor Titular de Tecnología Educativa de la Universidad de Sevilla, Director del Departamento de Didáctica y Organización educativa de la Universidad de Sevilla, Director del Grupo de Investigación Didáctica. Ha publicado diferentes trabajos en revistas especializadas sobre Tecnología Educativa. Ha impartido conferencias en diferentes Universidades españolas y latinoamericanas. Pueden encontrarse más referencias del autor en http://tecnologiaedu.us.es Correo electrónico de contacto: jbarroso@us.es 